\title{
Solitary fibrous tumor of the meninges
}

Sir,

A 30-year-old male presented with a history of headaches and vomiting for 2 weeks. He had bilateral papilledema. There were no focal neurological deficits. Computer tomography (CT) scan of the brain showed a contrast enhancing dural-based mass attached to the falx cerebri and extending on either side of the midline. Magnetic resonance imaging (MRI) of the brain showed the lesion to be a falx-based tumor, which was isointense on T1 and T2 weighted images [Figure 1]. He underwent a biparietal craniotomy, interhemispheric approach, and total excision of the tumor. At surgery, the tumor had a poor plane of dissection from the surrounding brain and was very vascular.

The histopathology showed a very cellular tumor that had spindle cells arranged in long fascicles with an intervening collagenous stroma. There was no evidence of mitosis or anaplasia. Immunohistochemistry showed that the cells were positive for CD34, bcl-2, and vimentin and negative for epithelial membrane antigen (EMA) and S-100 [Figure 2]. The MIB-1 labeling index averaged $2 \%$. Based on these findings, a diagnosis of solitary fibrous tumor was made. The patient made good neurological recovery and is symptom-free 1 year postoperatively. The follow-up CT scan shows no evidence of recurrent tumor.

Solitary fibrous tumors (SFT) are rare mesenchymal tumors arising from the meninges, which clinically and radiologically resemble meningiomas. ${ }^{[1]-[3]}$ Histopathologically they resemble fibrous meningiomas and haemangiopericytomas. ${ }^{[2],[4]}$ Solitary fibrous tumors have distinct immunohistochemical features, which distinguished them from meningiomas and

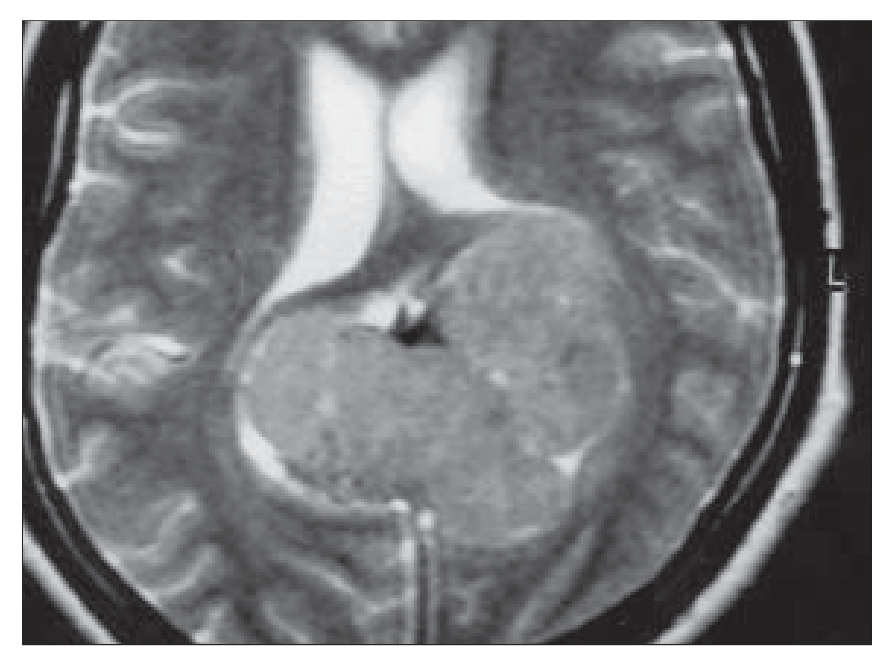

Figure 1: Axial T2 weighted MRI showing the falx based tumor 


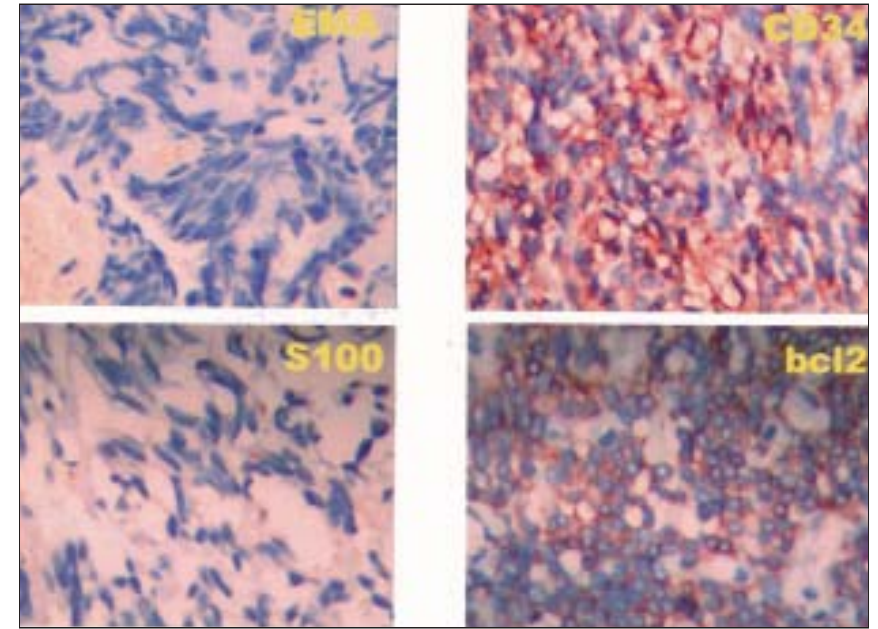

Figure 2: Immunohistochemistry showing strong positivity for CD34 and bcl2, while epithelial membrane antigen (EMA) and S-100 are negative

hemangiopericytomas. ${ }^{[2],[4]}$ These tumors follow a relatively benign course when compared to hemangiopericytomas. ${ }^{[2]}$ These tumors present as dural-based masses and have been reported to occur in both supra and infratentorial locations..$^{[2],[3]}$ Spinal SFTs are even rarer and can occur in both intra and extramedullary locations. ${ }^{[2],[3]}$

In the past, SFTs have been included along with meningiomas or hemangiopericytomas. ${ }^{[5]}$ Solitary fibrous tumors are cellular tumors that consist of spindle cells dispersed in fascicles. There is a characteristic eosinophilic collagenous stroma in which the spindle cells are present. ${ }^{[2],[3],[6]}$ The pathological spectrum extends from benign to anaplastic tumors. ${ }^{[2],[3]}$ MIB1 labeling indices range from 1 to $18 \%$. $^{[3],[6]}$

Immunohistochemistry of SFTs show positivity for CD34, bcl2, and vimentin and negativity for EMA and S-100. ${ }^{[2]-[6]}$ Strong CD34 and bcl2 positivity are characteristic immunohistochemical features of SFT. ${ }^{[2],[5]}$ In contrast, meningiomas show EMA positivity and hemangiopericytomas have a patchy and weak reactivity to CD34. ${ }^{[4]}$

The origin of these tumors has been controversial and both a mesothelial and mesenchymal origin have been hypothesized. ${ }^{[6]}$

The natural history of SFTs is largely unknown due to limited follow-up data. Most of the reports suggest a favorable outcome after complete resection and this suggests a benign behavior. ${ }^{[2],[3],[5]}$ Recurrences after subtotal excision have been reported. ${ }^{[2]}$ Extracranial metastases are rare. ${ }^{[7]}$ There is a recent report of meningeal SFT that progressed to malignant transformation and CSF dissemination over a course of repeated recurrences. ${ }^{[8]}$ The management of meningeal SFTs involves total excision followed by regular clinical and radiological follow up. In the rare cases of SFTs demonstrating anaplasia and mitosis, postoperative radiotherapy should be considered. Treatment options for residual and recurrent SFTs include surgery, radiotherapy, or stereotactic radiosurgery. ${ }^{[2],[8]}$

\section{Rahul Lath, Alok Ranjan, Swarnalata Gowrishankar $^{1}$}

Departments of Neurosurgery and 1Pathology, Apollo Hospitals, Jubilee Hills, Hyderabad, India. E-mail: rahullath@hotmail.com

\section{References}

1. Scheithauer PW. Mesenchymal, non-meningothelial tumors. In: Kleihaues P, Cavanee WK, editors. Pathology and Geneties of Tumours of the Nervous System. Lyon: IARC Press; 2000. p. 185-9.

2. Tihan T, Viglione M, Rosenblum MK, Olivi A, Burger PC. Solitary fibrous tumours in central nervous system. A clinicopathologic review of 18 cases and comparison to meningeal hemangiopericytomas. Arch Pathol Lab Med 2003;127:432-9.

3. Carneiro SS, Scheithauer BW, Nascimento AG, Hirose T, Davis DH. Solitary fibrous tumour of the meninges: A lesion distinct from fibrous meningioma. A clinicopathologic and immunohistochemical study. Am J Clin Pathol 1996;106:217-24.

4. Perry A, Scheithauer BW, Nascimento AG. The imunophenotypic spectrum of meningeal hemangiopericytoma: A comparison with fibrous meningioma and solitary fibrous tumour of the meninges. Am J Surg Pathol 1997;21:1354-60.

5. Suzuki SO, Fukui M, Nishio S, Iwaki T. Clinicopathological features of solitary fibrous tumour of the meninges: An immunohistochemical reappraisal of cases previously diagnosed to be fibrous meningioma or haemangiopericytoma. Pathol Int 2000;50:808-17

6. Prayson RA, McMahon JT, Barnett GH. Solitary fibrous tumour of the meninges. Case report and review of the literature. J Neurosurg 1997;86:1049-52.

7. Ogawa K, Tada T, Takahashi S, Sugiyama N, Inaguma S, Takahashi S, et al. Malignant solitary fibrous tumour of the meninges. Virchows Arch 2004:444:459-64

8. Miyashita K, Hayashi Y, Fujisawa H, Hasegawa M, Yamashita J. Recurrent intracranial solitary fibrous tumour with cerebrospinal fluid dissemination. J Neurosurg 2004;101:1045-8.

Accepted on 27-12-2004 